\title{
Evaluation of a local ICU sedation guideline on goal-directed administration of sedatives and analgesics
}

Jeremy R DeGrado'

Kevin E Anger'

Paul M Szumita'

Carol D Pierce ${ }^{2}$

Anthony $\mathrm{F}_{\text {Massaro }}{ }^{3}$

'Department of Pharmacy,

${ }^{2}$ Department of Nursing, ${ }^{3}$ Department of Pulmonary Medicine, Brigham and

Women's Hospital, Boston, MA, USA
Correspondence: Jeremy R DeGrado Department of Pharmacy, Brigham and Women's Hospital, 75 Francis St, Boston, MA 02II5, USA

Tel + I 6177327677

Fax + I 6175662396

Email jdegrado@partners.org
This article was published in the following Dove Press journal: Journal of Pain Research

29 April 2011

Number of times this article has been viewed

Purpose: Sedatives and analgesics are commonly used in mechanically ventilated patients in the intensive care unit. Sedation guidelines have been shown to improve sedation management as well as various patient outcomes. The main objective was to evaluate adherence to a sedation guideline with both sedative prescribing and documentation of Richmond Agitation-Sedation Scale (RASS) scores.

Methods: In a retrospective chart review, data was collected on 111 medical intensive care unit patients mechanically ventilated via endotracheal tube for 12 hours or greater at Brigham and Women's Hospital. Fifty-seven patients were evaluated pre-guideline implementation and 54 patients were evaluated post-guideline.

Results: Significant increases were seen in the post-guideline group in goal-directed sedation with a patient-specific RASS goal in the sedation order: 21.3 vs $85.4 \%(P<0.001)$, and mean number of sedation assessments per 24 hours using the RASS: 4.7 vs $11.4(P<0.001)$. Similarly, this group experienced a higher percentage of RASS scores at their sedation goal: 31.4 vs $44.1 \%(P<0.001)$. No difference was seen in other clinical endpoints.

Conclusion: Implementation and routine application of a hospital pain and sedation guideline was associated with significantly improved sedation metrics, such as goal-directed sedation, as well as frequency of sedation level assessment and documentation. An increase was observed in the time that post-guideline patients spent at or near their RASS goal.

Keywords: sedation, agitation, guideline, RASS, mechanically ventilated, intensive care unit

\section{Introduction}

Mechanically ventilated patients often receive both analgesic and sedative medications to reduce agitation, alleviate discomfort and pain, increase ventilator synchrony, decrease oxygen consumption, and ensure patient safety. ${ }^{1,2}$ The etiology of agitation in mechanically ventilated intensive care unit (ICU) patients can be linked to pain, delirium, sleep deprivation, alarms, tests and procedures, prolonged immobility, and other comorbidities. ${ }^{1,2}$ Sedatives and analgesics have adverse effects associated with them including oversedation, respiratory depression, hemodynamic instability, gastrointestinal effects, and drug accumulation. ${ }^{1-3}$ Utilization of sedatives and analgesics in the ICU has been associated with increased risk of delirium, duration of mechanical ventilation, ICU length of stay, and cost. ${ }^{1-12}$

Current guidelines for sedation and analgesia recommend titration of sedatives to a goal using a validated assessment tool to improve the quality of sedation therapy in the ICU. ${ }^{1,13}$ Despite demonstration of both the feasibility and benefits of implementing sedation assessment tools and guidelines, ${ }^{14-26}$ recent surveys indicate only 
about $60 \%-70 \%$ of institutions have a sedation guideline in place. ${ }^{27-29}$

In 2006, a guideline for the management of pain, sedation, and neuromuscular blockade was developed and implemented at Brigham and Women's Hospital. The Richmond Agitation-Sedation Scale (RASS), a 10-point, validated sedation scale, is the preferred sedation assessment tool recommended in the guideline. ${ }^{30,31}$ The objective of this retrospective analysis was to assess adherence with core guideline measures, including goal-directed sedation prescribing and sedation assessment using the RASS, and its association with patient outcomes.

\section{Patients and methods}

We conducted a retrospective analysis of patients receiving mechanical ventilation in the 20-bed medical intensive care unit (MICU) at Brigham and Women's Hospital from February to March 2006 (pre-guideline) and February to March 2007 (post-guideline). Approval was obtained from the Institutional Review Board before study initiation. All patients $\geq 18$ years old who were mechanically ventilated for $\geq 12$ hours during the two 2-month periods were assessed for inclusion in the analysis. Patients were excluded if they were mechanically ventilated for $<12$ hours or were receiving mechanical ventilation via a tracheostomy on admission to the ICU. Patients that received a tracheostomy during the study period were only followed until the procedure.

Baseline characteristics, including past medical history, vital signs over the first 24 hours of mechanical ventilation, critical labs, incidence of organ dysfunction, vasopressor requirement, and respiratory support were collected. Severity of illness of each patient group was estimated by the numbers of organ dysfunction in both groups. Organ dysfunction was defined using the following criteria: hematologic (platelet count $<80,000 /$ $\left.\mathrm{mm}^{3}\right)$, respiratory $\left(\mathrm{PaO} 2 / \mathrm{FiO}_{2}<300\right)$, renal (creatinine $\geq 1.5 \mathrm{mg} / \mathrm{dL}$ ), cardiovascular (average heart rate $>100 \mathrm{bpm}$ over first 24 hours or necessity of vasoactive agents), and hepatic (Tbili $\geq 1.2 \mathrm{mg} / \mathrm{dL}$ ). Critical medications were defined as continuous infusions of midazolam, lorazepam, propofol, dexmedetomidine, fentanyl, hydromorphone, insulin, and vasoactive agents.

\section{Guideline information}

In 2006, a multidisciplinary team of physicians, nurses, and pharmacists developed and implemented a 20-page hospital-wide guideline for the management of pain, agitation, and neuromuscular blockade at our institution. The guideline provides core recommendations regarding medication selection and dosing, assessment of pain, sedation, delirium, paralysis, and use of daily interruption strategies. The guideline was implemented hospital-wide with education provided to all ICU nurses, physicians, and pharmacists at baseline. Educational sessions were provided every month to new physicians rotating through the ICU. Continuous education was also provided to ICU nurses and reinforced through annual competency training.

The guideline provides recommendations on assessment tools for sedation, analgesia, and neuromuscular blockade. Patients are assessed for pain using a numerical and/or visual analog scale if possible, and then for other etiologies of agitation. Sedation should be assessed, via the RASS score, and documented at least once every 2 hours while patients are mechanically ventilated. The guideline recommends a goal RASS score of " 0 to -1 " for most patients, although specific exceptions exist (ie, neuromuscular blockade).

The guideline also provides education and recommendations on drug administration and dosing for sedatives, analgesics, and antipsychotic agents. As per the guideline, nurses are able to administer and titrate analgesics and sedatives by either boluses or, if necessary, continuous infusions to the patient's goal RASS score. Recommended sedatives include midazolam, propofol, lorazepam, diazepam, and dexmedetomidine. Propofol is the first-line agent in patients who meet criteria for head injury or frequent awakening for neurological assessment. Midazolam is considered first-line for patients who do not meet these criteria, with propofol, lorazepam, diazepam, and dexmedetomidine being second- and third-line agents. Recommended analgesics include fentanyl, morphine, and hydromorphone. Fentanyl is the preferred analgesic, especially in hemodynamically unstable patients, with hydromorphone and morphine being second-line therapies. Use of bolus dosing is recommended for select analgesics and sedatives before initiation and upward titration of continuous infusions. Recommended antipsychotics include haloperidol, quetiapine, and olanzapine. Incorporation of daily interruption strategies is recommended in patients not meeting exclusion criteria set forth by the guideline.

Simultaneously with guideline implementation, a change was implemented within the computerized provider order entry (CPOE) system. A field containing, "titrate to RASS =" was programmed to auto-populate in all orders for continuous sedation. Providers were able to enter a patient-specific RASS goal within the sedation order but could also leave this blank if they did not wish to specify a goal. 


\section{Endpoints}

The two primary endpoints were the frequency of documentation of a RASS goal in orders for continuous infusions of sedatives as well as the frequency of assessment and documentation of patients' RASS scores by the nurses. Secondary endpoints included time that the patient spent at or within one point of their RASS goal, time to initial RASS documentation, ICU and hospital length of stay, hospital mortality duration of mechanical ventilation, amount of sedatives and analgesics administered, and number of tracheostomies, reintubations, and head computerized tomography or magnetic resonance imaging scans while patients were in the ICU.

\section{Statistical analyses}

Discrete data are presented as the number and percentage of the occurrence of the variable. Continuous data are presented as means \pm standard deviation (SD) for normally distributed variables, and medians for non-normally distributed variables. The Mantel Haenszel Chi Square test was used to compare categorical data between groups. The twoSided $t$-test was used to compare continuous parametric data while the Mann-Whitney $U$-test was used for nonparametric data. All statistical tests were two-tailed and used a cutoff of $\alpha<0.05$ for statistical significance.

\section{Results}

One hundred eighty-one patients receiving mechanical ventilation from February to March 2006 and February to March 2007 were screened. Seventy were excluded (49 due to intubation $<12$ hour, 20 were receiving mechanical ventilation via tracheostomy, and one due to transfer to surgical ICU within 18 hours of admission). Therefore, $111(66 \%)$ were included in the retrospective analysis: 57 patients in the pre-guideline group and 54 patients in the post-guideline group. Baseline demographics (Table 1) were significantly different between the two groups with higher mean vasoactive agent requirements $(0.39$ vs 0.76 ; $P=0.03)$, mean organ dysfunctions (1.8 vs $2.2 ; P=0.04)$, percentage of patients requiring two or more vasoactive agents $(5.3 \%$ vs $22.2 \% ; P=0.009)$, percentage of patients with three or more organ dysfunctions $(22.8 \%$ vs $44.4 \%$; $P=0.02)$, and lower MAP (82.6 vs $75.4 \mathrm{mmHg} ; P=0.004)$ in the post-guideline group.

Table I Baseline demographics

\begin{tabular}{|c|c|c|c|}
\hline Variable & $\begin{array}{l}\text { Pre-guideline } \\
\mathrm{n}=57\end{array}$ & $\begin{array}{l}\text { Post-guideline } \\
\mathrm{n}=54\end{array}$ & P-value \\
\hline Age, mean $\pm S D$, years & $63.3 \pm 17.2$ & $62.3 \pm 16.5$ & 0.83 \\
\hline Male, no. (\%) & $25(43.9)$ & $26(48.1)$ & 0.65 \\
\hline $\mathrm{MAP}$, mean $\pm \mathrm{SD}, \mathrm{mm} \mathrm{Hg}$ & $82.6 \pm 12.7$ & $75.4 \pm 12.8$ & 0.004 \\
\hline $\begin{array}{l}\text { No. of vasoactive medications on admission per } \\
\text { patient, mean } \pm S D\end{array}$ & $0.39 \pm 0.7$ & $0.76 \pm 1$ & 0.031 \\
\hline Patients with $\geq 2$ vasoactive agents, no. (\%) & $3(5.3)$ & $12(22.2)$ & 0.009 \\
\hline No. organ dysfunction per patient, mean \pm SD & $1.8 \pm 0.9$ & $2.2 \pm 1.1$ & 0.037 \\
\hline Patients with $\geq 3$ organ dysfunction, no. (\%) & $13(22.8)$ & $24(44.4)$ & 0.016 \\
\hline \multicolumn{4}{|l|}{ Organ dysfunction, no. (\%) } \\
\hline Cardiac & $33(57.9)$ & $39(72.2)$ & 0.11 \\
\hline Hematologic & $3(5.3)$ & $8(14.8)$ & 0.09 \\
\hline Hepatic & $12(2 \mid .1)$ & I5 (27.8) & 0.41 \\
\hline Renal & $26(45.6)$ & $26(48.1)$ & 0.79 \\
\hline Respiratory & $30(52.6)$ & $33(61.1)$ & 0.56 \\
\hline $\mathrm{SCr}$, mean $\pm \mathrm{SD}, \mathrm{mg} / \mathrm{dL}$ & $2.0 \pm 2.1$ & $2.5 \pm 2.7$ & 0.29 \\
\hline WBC, mean $\pm S D, \times 10^{3} /$ microliter & $12.9 \pm 6.2$ & $13.5 \pm 8.3$ & 0.67 \\
\hline \multicolumn{4}{|l|}{ PMH, no. (\%) } \\
\hline DM & $9(15.8)$ & $12(22.2)$ & 0.39 \\
\hline COPD/Asthma & $18(31.6)$ & $12(22.2)$ & 0.27 \\
\hline Hypertension & $23(40.4)$ & $14(25.9)$ & 0.11 \\
\hline CAD & $13(22.8)$ & $16(29.6)$ & 0.63 \\
\hline CHF/Cardiomyopathy & $13(22.8)$ & $5(9.3)$ & 0.05 \\
\hline Atrial fibrillation & $9(15.8)$ & $10(18.5)$ & 0.58 \\
\hline CRI/ESRD & II (19.3) & $10(18.5)$ & 0.71 \\
\hline Cancer & $14(24.6)$ & $17(31.5)$ & 0.65 \\
\hline
\end{tabular}

Abbreviations: CAD, coronary artery disease; CHF, congestive heart failure; COPD, chronic obstructive pulmonary disease; CRI, chronic renal insufficiency; DM, diabetes mellitus; ESRD, end stage renal disease; PMH, past medical history. 
Table 2 Sedation metrics

\begin{tabular}{|c|c|c|c|}
\hline Variable & $\begin{array}{l}\text { Pre-guideline } \\
\mathrm{n}=\mathbf{5 7}\end{array}$ & $\begin{array}{l}\text { Post-guideline } \\
n=54\end{array}$ & P-value \\
\hline No. of continuous intravenous sedation orders & 89 & 89 & 0.69 \\
\hline Sedation orders with RASS goal, no. (\%) & $19(21.3)$ & $76(85.4)$ & $<0.001$ \\
\hline Total $n r$ of RASS scores during sedation & 1078 & 2762 & 0.002 \\
\hline No. of RASS per 24 hour sedation, mean \pm SD & $4.7 \pm 4.9$ & $11.4 \pm 2.9$ & $<0.001$ \\
\hline RASS scores at goal, \% & 31.4 & 44.1 & $<0.001$ \\
\hline RASS scores within I of goal, $\%$ & 45.1 & 66.7 & $<0.001$ \\
\hline Time to initial RASS documentation, mean $\pm S D$, hour & $24.5 \pm 39.1$ & $2.4 \pm 5.5$ & $<0.001$ \\
\hline
\end{tabular}

Abbreviation: RASS, Richmond Agitation-Sedation Scale.

\section{Primary endpoints}

Primary outcomes are presented in Table 2. There were 89 orders for continuous sedation in each group. In terms of continuous analgesia, there were 57 orders in the pre-guideline group and 65 orders in the post-guideline group. A significant increase was seen post-guideline in the percentage of sedatives $(21.3 \%$ vs $85.4 \% ; P<0.001)$ and analgesics ( 15.8 vs $55.4 \% ; P<0.001)$ ordered with a RASS goal. The total number of RASS assessments documented while patients were sedated was higher in the postguideline group (Table 2). A significant difference favoring the post-guideline group was seen in the average number of RASS assessments per 24-hour period of sedation (Figure 1). The median number of daily RASS assessments per patient (Figure 2) was significantly higher for the majority of days in the post-guideline group.

\section{Secondary endpoints}

In patients in the post-guideline group, those with a prespecified RASS goal experienced an increase in the percentage of RASS scores at goal $(31.3 \%$ vs $44.1 \% ; P<0.001)$ and the number of scores within one point of goal ( $45.1 \%$ vs $66.7 \%$; $P<0.001$ ) (Figure 3). Fifty-four percent (54\%) of sedation orders had a RASS goal of 0 to -1 while only $5 \%$ of orders

\section{RASS assessment documentation}

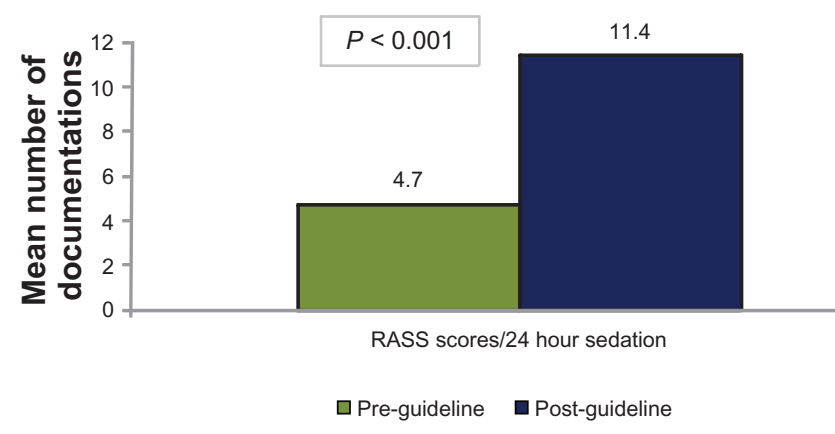

Figure I RASS assessments.

Abbreviation: RASS, Richmond Agitation-Sedation Scale. had a RASS goal deeper than -2 . Time to initial RASS assessment and documentation in the post-guideline group was significantly shorter ( 24.5 hours vs 2.4 hours; $P<0.001$ ) in the post-guideline group.

Our study was not powered to detect differences in our secondary outcome parameters and, accordingly, no difference was seen between the pre- and post-guideline groups in mean ICU or hospital length of stay hospital mortality, duration of mechanical ventilation, number of reintubations, or number of head CT scans or MRIs performed after the initial 48 hours in the ICU. Midazolam (75\% vs $78 \%$ patients; $P=0.96)$ and fentanyl ( $77 \%$ vs $81 \% ; P=0.60)$ were the most frequently prescribed sedative and analgesic in both the pre- and post-guideline groups, respectively. No difference was seen between the duration and amount of continuous analgesia and sedation among the two cohorts.

\section{Discussion}

In our retrospective cohort analysis, we found the implementation of a hospital-wide guideline for the management of pain, agitation, and neuromuscular blockade increased utilization of goal-directed administration of sedatives and frequency of sedation assessment documentation in a mechanically ventilated medical ICU population. Post-guideline improvements in sedation metrics were also demonstrated by faster time to initial sedation assessment and a higher percentage of RASS assessments at goal or within one point of goal.

Current sedation and analgesia guidelines recommend the use of sedation guidelines or protocols as well as titration of sedative dose to a defined endpoint. ${ }^{1}$ No guideline or protocol has been established as superior in the literature, and adaptation of other institutional protocols or guidelines can be difficult. Clinicians seeking to improve sedation therapy should develop and implement local guidelines or protocols that fit the culture, resources, and medication formulary of their institution. ${ }^{32}$ Our study constitutes a quality improvement project to assess the impact of a local guideline on sedation 


\section{Daily RASS assessments per patient}

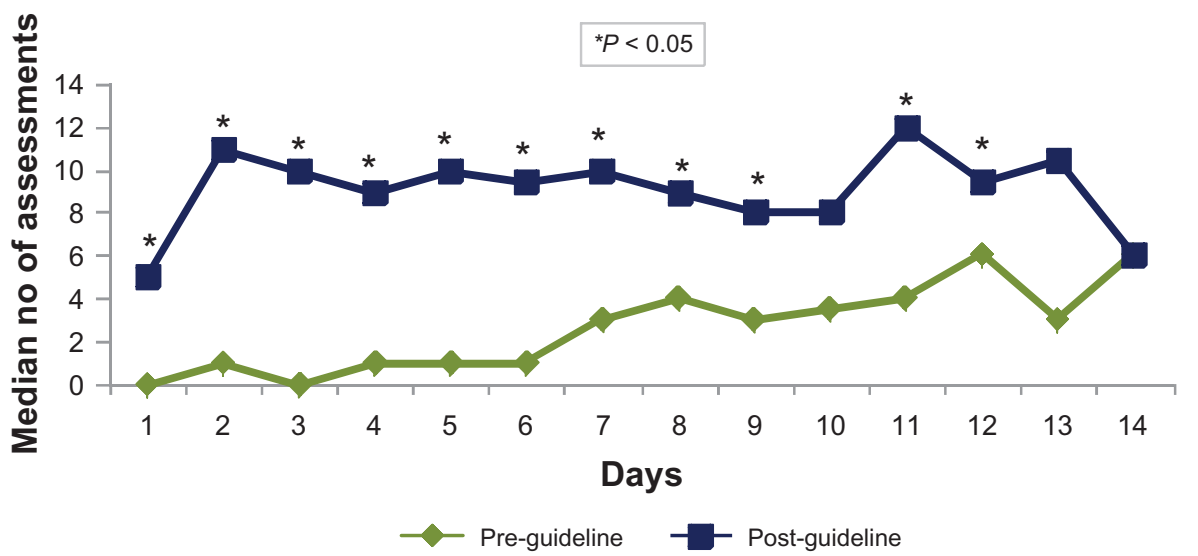

No.of patients
Pre-guideline
Post-guideline

$\begin{array}{lllll}57 & 56 & 43 & 30 & 26\end{array}$

$\begin{array}{lllll}54 & 54 & 46 & 37 & 31\end{array}$

$\begin{array}{lllllllll}21 & 20 & 15 & 11 & 10 & 10 & 9 & 9 & 8\end{array}$

$\begin{array}{lllllllll}28 & 24 & 21 & 21 & 16 & 11 & 10 & 10 & 10\end{array}$

Figure 2 RASS Assessments.

Abbreviation: RASS, Richmond Agitation-Sedation Scale.

therapy in a mechanically ventilated medical intensive care unit population. Continuous quality assessment is needed to ensure adherence to guidelines or protocols, and to develop initiatives to improve adherence and outcomes. ${ }^{17,18}$ Analysis of metrics are needed to assess the impact of guideline and protocolized care, however establishment of ideal sedation metrics is not defined in the literature. ${ }^{33}$ Published sedation improvement initiatives provide an array of sedation metrics used to validate the impact of the intervention on patient outcomes. ${ }^{17,24,33}$ In our analysis, the percentage of RASS assessments at (44\%) or near goal (67\%) was significantly higher post-guideline. This percentage is lower than reports from recent studies, however it should be noted that larger target sedation ranges were used. ${ }^{34,35}$

\section{Achievement of sedation goal}

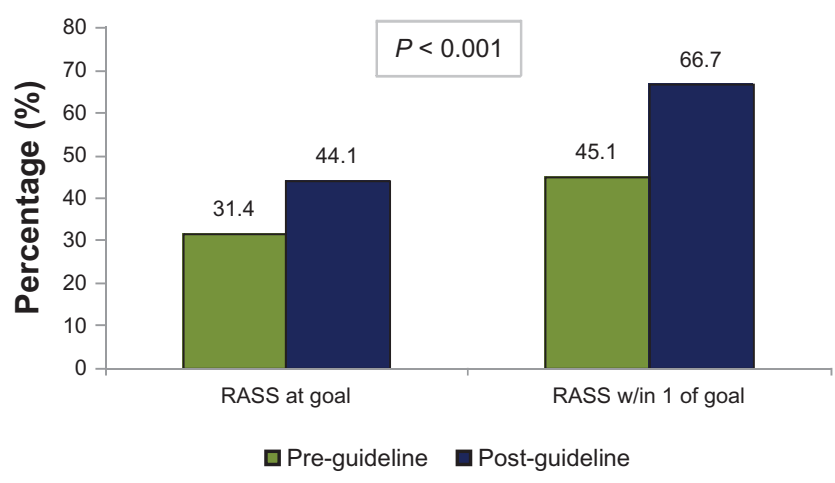

Figure 3 Sedation outcomes. Abbreviation: RASS, Richmond Agitation-Sedation Scale.
Several analyses are published in the literature examining the effects of different sedation related practices on outcomes in mechanically ventilated ICU patient populations. ${ }^{14-26}$ In 1999, Brook and colleagues randomized 321 mechanically ventilated medical ICU patients to receive sedation by either a nursing-implemented protocol or by a non-protocol approach. ${ }^{14}$ The protocol allowed for nurses to administer and titrate analgesics and sedatives by either boluses or continuous infusions to a pre-determined Ramsay score. Protocolized patients experienced a shorter duration of mechanical ventilation (89.1 vs 124 hours; $P=0.003$ ), decreased ICU and hospital length of stay (5.7 vs 7.5 days; $P=0.013$, and 14.0 vs 19.9 days; $P<0.001$, respectively), and a lower requirement for tracheostomy $(6.2 \%$ vs $13.2 \% ; P=0.038)$. Patients getting non-protocol directed sedation had an increased duration of continuous infusion sedative therapy (3.5 vs 5.6 days; $P=0.003$ ), whereas protocol-directed sedation was determined to be an independent variable associated with successful weaning from mechanical ventilation (RR 1.37; $P=0.026$ ). Brook and colleagues demonstrated clinically significant reductions in many clinical outcomes, but did not comprehensively evaluate the impact of sedation related metrics on their findings. ${ }^{14}$

In 2006, Chanques and colleagues conducted a before-after study to examine the impact of systematic assessment of pain and agitation in 230 surgical and medical ICU patients. ${ }^{19}$ Patients were assessed twice daily for pain using the numerical rating scale (NRS) or behavioral pain scale (BPS), and for sedation using the RASS. The post 
intervention group demonstrated more RASS assessments per patient per day ( 1.3 vs $1.6 ; P<0.001)$, fewer incidence of both pain and agitation $(63 \%$ vs $42 \% ; P=0.002$, and $29 \%$ vs $12 \% ; P=0.002$, respectively), reduced incidence of nosocomial infection ( $8 \%$ vs $17 \% ; P=0.01)$, and reduced duration of mechanical ventilation (120 vs 65 hours; $P=0.01)$. Despite a minimal increase in the frequency of sedation assessment, Chanques and colleagues described a lower incidence of several clinical outcomes. The optimal frequency of sedation assessment has yet to be determined in the literature and current guidelines do not provide recommendations. ${ }^{1,17,33}$ Our guideline recommends sedation assessments every 2 hours. More frequent assessment of RASS in our post-guideline cohort did not demonstrate improvement in clinical outcomes; however differences in sample size, sedative agent choices, and severity of illness amongst our cohort groups likely prevented us from seeing the differences demonstrated by Brook and colleagues and Chanques and colleagues.

Analysis of sedation improvement initiatives have shown various benefits and positive associations, such as decreased time of mechanical ventilation, time in the ICU, incidence of pain and agitation, incidence of nosocomial infections, amount of sedatives and analgesics administered, cost, and increased sedation assessments. ${ }^{14-26}$ Despite improvements in various sedation metrics, implementation of the guideline was not associated with improvement in clinical outcomes such as mortality, length of stay, and duration of mechanical ventilation. While improvement in clinical outcomes is ideal, our study was underpowered to detect these and should be viewed as a sedation metrics assessment.

Our study has several limitations that may have inhibited our ability to assess metrics and outcomes of guideline implementation in our cohort. First, our study was not prospective or randomized; however the analysis was performed using a sequential study of two time periods in which all consecutive patients were screened for enrollment. Second, due to retrospective design, documentation, and information availability, we were unable to evaluate cost considerations and incidence of nosocomial infections. Also, differences in the number of vasopressors and organ dysfunctions at baseline suggest our post-guideline group had a higher severity of illness. These significant differences may have resulted in our inability to detect improvement in clinical outcomes in the post-guideline cohort. Finally, in addition to the implementation of a sedation guideline, a CPOE intervention was made for providers to enter a patient-specific RASS goal. This intervention did not require providers to enter a RASS goal, but may have contributed to the increase in patient-specific RASS goals seen in the sedative orders.

\section{Conclusions}

The implementation of a guideline for the management of pain and sedation was associated with increased utilization of goal-directed administration of sedatives, frequency of sedation assessment documentation, and percentage of assessments at or near goal in a mechanically ventilated medical ICU population; however these changes were not associated with improvement in clinical outcomes. The results could be attributable to differences in the severity of illness between the cohorts and sample size needed to evaluate specific outcome variables. Further analysis is needed to evaluate sedation guideline interventions and metrics that correlate with improvement in clinical outcomes.

\section{Disclosure}

This study was performed at Brigham and Women's Hospital and was presented in part at the 2009 annual meeting of the Society of Critical Care Medicine in Nashville, TN. No financial support was obtained for this analysis.

\section{References}

1. Jacobi J, Fraser GL, Coursin DB, et al. Clinical practice guidelines for the sustained use of sedatives and analgesics in the critically ill adult. Crit Care Med. 2002;30:119-141.

2. Sessler CN, Varney K. Patient-focused sedation and analgesia in the ICU. Chest. 2008;133:552-565.

3. Riker RR, Fraser GL. Adverse events associated with sedatives, analgesics, and other drugs that provide patient comfort in the intensive care unit. Pharmacotherapy. 2005;25:8S-18S.

4. Pandharipande P, Shintani A, Peterson J, et al. Lorazepam is an independent risk factor for transitioning to delirium in intensive care unit patients. Anesthesiology. 2006;104:21-26.

5. Ely EW, Shintani A, Truman B, et al. Delirium as a predictor of mortality in mechanically ventilated patients in the intensive care unit. JAMA. 2004;291:1753-1762.

6. Ostermann ME, Keenan SP, Seiferling RA, et al. Sedation in the intensive care unit: a systematic review. JAMA. 2000;283:1451-1459.

7. Rello J, Diaz E, Roque M, et al. Risk factors for developing pneumonia within 48 hours of intubation. Am J Respir Crit Care Med. 1999;159: $1742-1746$.

8. Kollef MH, Leavy NT, Ahrens TS, et al. The use of continuous iv sedation is associated with prolongation of mechanical ventilation. Chest. 1998;114:541-548.

9. Arroliga A, Frutos-Vivar F, Hall J, et al. Use of sedatives and neuromuscular blockade in a cohort of patients receiving mechanical ventilation. Chest. 2005;128:496-506.

10. Kress JP, Pohlman AS, Hall JB. Sedation and analgesia in the intensive care unit. Am J Respir Crit Care Med. 2002;166:1024-1028.

11. Malacrida R, Fritz ME, Suter PM, et al. Pharmacokinetics of midazolam administered by continuous intravenous infusion to intensive care patients. Crit Care Med. 1992;20:1123-1126.

12. Szumita PM, Baroletti SA, Anger KE, et al. Sedation and analgesia in the intensive care unit: evaluating the role of dexmedetomidine. Am J Health-Syst Pharm. 2007;64:37-44. 
13. Dellinger RP, Levy MM, Carlet JM, et al. Surviving Sepsis Campaign: international guidelines for management of severe sepsis and septic shock: 2008. Crit Care Med. 2008;36:296-316.

14. Brook AD, Ahrens TS, Schaiff R, et al. Effect of a nurse-implemented sedation protocol on the duration of mechanical ventilation. Crit Care Med. 1999;27:2609-2615.

15. Kress JP, Pohlman AS, O'Connor MF, et al. Daily interruption of sedative infusions in critically ill patients undergoing mechanical ventilation. N Engl J Med. 2000;342:1471-1477.

16. Girard TD, Kress JP, Fuchs BD, et al. Efficacy and safety of paired sedation and ventilator weaning protocol for mechanically ventilated patients in an intensive care unit (Awakening and Breathing Controlled trial): a randomized controlled trial. Lancet. 2008;371:126-134.

17. Pun BT, Gordon SM, Peterson JF, et al. Large-scale implementation of sedation and delirium monitoring in the intensive care unit: a report from two medical centers. Crit Care Med. 2005;33:1199-1205.

18. Mehta S, Burry L, Martinez-Motta JC, et al. A randomized trial of daily awakening in critically ill patients managed with a sedation protocol. Crit Care Med. 2008;36:2092-2099.

19. Chanques G, Jaber S, Barbotte E, et al. Impact of systemic evaluation of pain and agitation in an intensive care unit. Crit Care Med. 2006;34: 1691-1699.

20. Marshall J, Finn CA, Theodore AC. Impact of a clinical pharmacistenforced intensive care unit sedation protocol on duration of mechanical ventilation and hospital stay. Crit Care Med. 2008;36:427-433.

21. MacLaren R, Plamondon JM, Ramsay KB, et al. A prospective evaluation of empiric versus protocol-based sedation and analgesia. Pharmacotherapy. 2000;20:662-672.

22. Mascia MF, Koch M, Medicis JJ. Pharmacoeconomic impact of rational use of guidelines on the provision of analgesia, sedation, and neuromuscular blockade in critical care. Crit Care Med. 2000;28:2300-2306.

23. De Jonghe B, Bastuji-Garin S, Fangio P, et al. Sedation algorithm in critically ill patients without acute brain injury. Crit Care Med. 2005;33: 120-127.

24. Brattebo G, Hofoss D, Flaatten H, et al. Effect of a scoring system and protocol for sedation on duration of patients' need for ventilator support in a surgical intensive care unit. BMJ. 2002;324:1386-1389.
25. Quenot JP, Ladoire S, Devoucoux F, et al. Effect of a nurse-implemented sedation protocol on the incidence of ventilator-associated pneumonia. Crit Care Med. 2007;35:2031-2036.

26. Arias-Rivera S, del Mar Sanchez-Sanchez M, Santos-Diaz R, et al. Effect of a nursing-implemented sedation protocol on weaning outcome. Crit Care Med. 2008;36:2054-2060.

27. Mehta S, Burry L, Martinez-Motta JC, et al. Canadian survey of the use of sedatives, analgesics, and neuromuscular blocking agents in critically ill patients. Crit Care Med. 2006;34:374-380.

28. Tanios MA, de Wit M, Epstein SK, et al. Perceived barriers to the use of sedation protocols and daily sedation interruption: a multidisciplinary survey. J Crit Care. 2009;24:66-73.

29. Payen JF, Chanques G, Mantz J, et al. Current practices in sedation and analgesia for mechanically ventilated critically ill patients. Anesthesiology. 2007;106:687-695.

30. Sessler CN, Gosnell MS, Grap MJ, et al. The Richmond Agitation-Sedation Scale: validity and reliability in adult intensive care unit patients. Am J Resp Crit Care Med. 2002;166:1338-1344.

31. Ely EW, Truman B, Shintani A, et al. Monitoring sedation status over time in ICU patients: reliability and validity of the Richmond Agitation-Sedation Scale (RASS). JAMA. 2003;289:2983-2991.

32. Davis DA, Taylor-Vaisey A. Translating guidelines into practice: a systematic review of theoretic concepts, practical experience and research evidence in the adoption of clinical practice guidelines. Can Med Assoc J. 1997;157:408-416.

33. Weinert CR, Calvin AD. Epidemiology of sedation and sedation adequacy for mechanically ventilated patients in a medical and surgical intensive care unit. Crit Care Med. 2007;35:393-401.

34. Ruokonen E, Parviainen I, Jacob SM, et al. Dexmedetomidine versus propofol/midazolam for long-term sedation during mechanical ventilation. Intensive Care Med. 2009;35:282-290.

35. Riker RR, Shehabi Y, Bokesch PM, et al. Dexmedetomidine vs midazolam for sedation of critically ill patients: a randomized trial. JAMA 2009;301:489-499. 


\section{Appendix}

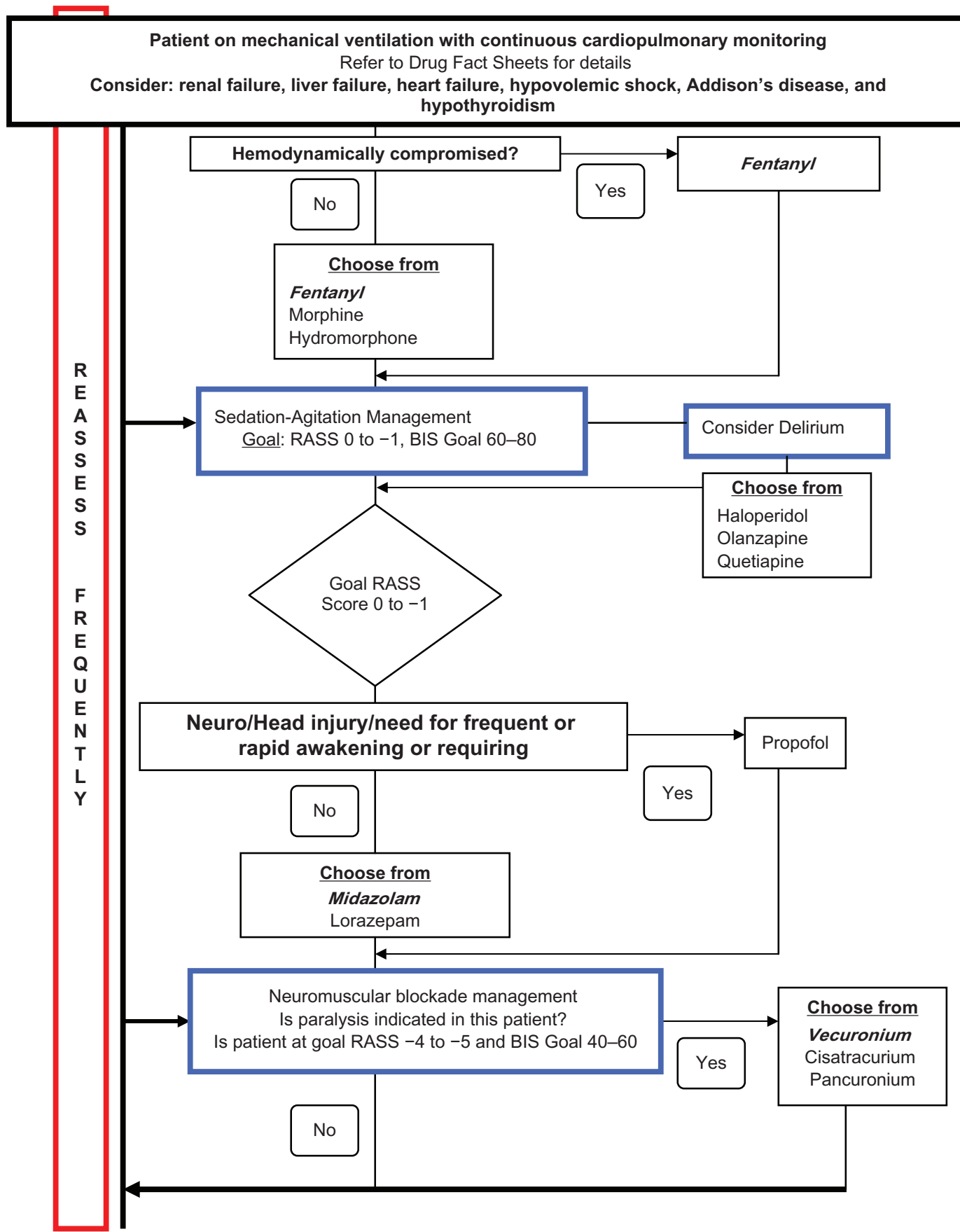

Appendix I Sedation guideline.

Journal of Pain Research

\section{Publish your work in this journal}

The Journal of Pain Research is an international, peer-reviewed, open access, online journal that welcomes laboratory and clinical findings in the fields of pain research and the prevention and management of pain. Original research, reviews, symposium reports, hypothesis formation and commentaries are all considered for publication.
The manuscript management system is completely online and includes a very quick and fair peer-review system, which is all easy to use. Visit http://www.dovepress.com/testimonials.php to read real quotes from published authors.

Submit your manuscript here: http://www.dovepress.com/journal-of-pain-research-journal 